\title{
A emergência das primeiras palavras: aspectos da produção sonora inicial da criança
}

\section{The emergency of first words: aspects of the child's initial sound production}

Mestre e Doutora em Estudos Linguísticos pela UFMG. Professora Adjunta da Faculdade de GUIMARÄES, D. M. L. Beyond early words: word template development in Brazilian Portuguese. The Emergence of phonology: whole word approaches and cross-linguistic evidence. Ted.London: 3. v. p. 291-316. E-mail: daniolive@yahoo.com

\section{Daniela Mara Lima Oliveira Guimarães}

RESUMO: Este estudo tem por objetivo discutir aspectos fonológicos da emergência da fala em crianças, em fase inicial de aquisição da linguagem a partir de um estudo longitudinal. Avaliam-se as características fonéticas da produção inicial de quatro crianças, a partir da teoria da Whole-Word Phonology (VIHMAN, 1996), procurando-se discutir quais os padrões motores são usados inicialmente na produção das primeiras palavras. 0 corpus para esta pesquisa foi obtido a partir da fala espontânea e da nomeação de figuras. A análise dos dados aponta que as formas das primeiras palavras podem ser variáveis para diferentes indivíduos. Foi possível observar a presença de padrões sonoros como CVCV, como uma espécie de resquício do balbucio, a ocorrência de palavras selecionadas do alvo adulto e, em outros sujeitos, a variabilidade, na produção inicial, ocorreu com a tentativa de uso de formas diversas e complexas. Os resultados indicam que a forma sonora inicial das primeiras palavras é variável e aponta caminhos possíveis de aquisição individual.

Palavras-chave: Aquisição fonológica; Padrões sonoros de palavras; Primeiras palavras.

ABSTRACT: This study aims to discuss phonological aspects of speech emergence in children, considering a longitudinal study. The phonetic characteristics of the initial production of four children are evaluated, considering Whole-Word Phonology theory (VIHMAN, 1996). It is discussed which motor patterns are used to produce the first words. The analysis of the data indicates that the forms of the first words can be variable for different individuals. Among them, we observed the presence of sound patterns as CVCV, as a remnant of the babble, the presence of selected words forms from the adult target and, in other subjects, the variability in the initial production and the attempt to produce diverse and complex forms. The results indicate that the initial sound form of the first words are variable and they point to possible paths of individual acquisition.

Keywords: Phonological acquisition; Child word templates; Early words. 


\section{Introdução}

A aquisição da fala é um período que, por sua especificidade e importância no desenvolvimento, tem chamado a atenção de vários pesquisadores envolvidos na compreensão da linguagem. Dentre os fatos que envolvem este período, a aquisição fonológica inicial coloca-se como um ponto relevante de discussão. No português brasileiro, há importantes trabalhos sobre a aquisição fonológica, os quais evidenciam, sobretudo, os segmentos que as crianças produzem, os processos fonológicos aplicados, a faixa etária de produção e a evolução na produção fonética ao longo dos anos (LAMPRECHT, et al., 2004). Tais trabalhos têm contribuído para melhor compreensão deste período e levantado pontos fundamentais sobre as teorias fonológicas.

Dentre os trabalhos realizados, há necessidade de pesquisas sobre a produção inicial, uma vez que, provavelmente pela dificuldade de obtenção de dados de fala, este período é, por vezes, obscurecido (OLIVEIRAGUIMARÃES, 2008). A produção inicial é compreendida, neste artigo, como período de emergência das primeiras palavras. Argumenta-se que o melhor conhecimento dos dados de emergência podem iluminar fatos que ocorrem posteriormente no desenvolvimento e, além disso, contribuir para uma visão mais clara do percurso de desenvolvimento infantil, identificando o que a criança é capaz de produzir e as estratégias que utiliza já no início do seu desenvolvimento.

Sendo assim, e considerando-se a perspectiva da Whole-Word-Phonology, a qual postula que a aquisição fonológica tem base em padrões que emergem do léxico, analisam-se, neste artigo, dados de produção inicial de palavras de quatro crianças em um estudo longitudinal. Todas as crianças gravadas produziam à época da primeira coleta uma palavra por enunciado e tinham a idade entre 1;6 a 2;00 anos, no início da gravação. A partir desses dados, procuram-se avaliar: a) a forma fonética das primeiras palavras; b) a relação entre tais formas e os padrões de balbucio possíveis; c) a aplicação de "moldes"ou templates para adaptação de tais palavras a um padrão fonológico e motor; d) as diferentes formas e os diferentes caminhos que as crianças percorrem na produção inicial.

Considerando-se tais objetivos, apresentam-se, nas próximas seções, os estudos sobre o balbucio e as primeiras palavras. Em seguida, analisam-se os dados e discutem-se os resultados.

\section{Antes das primeiras palavras: o balbucio e suas transições}

As primeiras manifestações sonoras das crianças constituem-se de vocalizações repetidas, além do choro e do riso. Estes surgem bem cedo no desenvolvimento e estabelecem um dos primeiros contatos do bebê com o mundo. Através do choro e do riso, as crianças pedem, confirmam, agradam, recusam, demonstram dor, fome e vão, assim, pouco a pouco, construindo o seu lugar no meio em que vivem. Além do choro e do riso, as manifestações vocais primárias constituem-se da produção involuntária de alguns sons e da manipulação de traços prosódicos, como a melodia, por exemplo (VIHMAN, 1996). Por volta dos 6 meses de idade (BAUMANWAENGLER, 1994; JUSCZYK, 1997), a criança começa a produzir o que se chama de balbucio. É neste momento que se percebe, de forma mais nítida, a produção de sílabas. Embora haja por parte dos cuidadores e daqueles que convivem com a criança a intenção de interpretação do balbucio, sabe-se que é um período de constante tentativa e amadurecimento motor através de movimentos repetitivos e que, a princípio, não carrega significado específico.

Tradicionalmente, o balbucio é classificado como reduplicado ou variado (este último também conhecido como não-reduplicado). 0 balbucio reduplicado ocorre quando a criança começa a produzir sílabas reduplicadas, geralmente, consistindo da sequência de uma consoante oclusiva e uma vogal 
central aberta, por exemplo, [bababa] (JUSCZYK, 1997, p. 174). O balbucio variado ocorre, em geral, após o balbucio reduplicado, embora, conforme demonstra Vihman (1996), pode haver sobreposição entre esses dois períodos. O balbucio variado é caracterizado pela alternância dos segmentos consonantais e vocálicos nas sequências de sílabas. Um exemplo de balbucio variado seria [maba].

O balbucio tem gerado extensas discussões teóricas. De acordo com Jakobson (1971), tal período tem pouca relação com o desenvolvimento fonológico e com a produção das primeiras palavras. É a chamada hipótese da descontinuidade. A evidência para a descontinuidade encontra-se em pesquisas as quais apontam que, após o balbucio, há um período de silêncio, que precede a produção das primeiras palavras. Além do período de silêncio, outra evidência utilizada para reforçar a hipótese da descontinuidade é a capacidade de a criança de produzir os mais variados sons durante o balbucio, os quais posteriormente desaparecem, com o advento das primeiras palavras. Conforme Jakobson (1971, p. 76):

Durante o estágio do balbucio (période du babil), a criança produz os mais variados sons com facilidade (por exemplo, cliques e palatalizados, arredondados, africados, sibilantes, consoantes uvulares etc) quase todos eliminados quando a criança procede ao estágio das primeiras palavras

(...) isto é, quando adota o primeiro valor semântico. (Tradução livre) ${ }^{1}$

Estudos posteriores, no entanto, refutam a hipótese de Jakobson sobre a descontinuidade e argumentam que o balbucio é um período preparatório para o desenvolvimento fonológico: é a chamada hipótese da continuidade. Vihman (1996) observa que alguns sons, como as consoantes silábicas, as vibrantes bilabiais e as consoantes labio-linguais, que ocorrem no balbucio,

${ }_{1}^{1}$ During babbling stage (période du babil) the child produces the most varied sounds with ease (for example
clicks and palatalised, rounded, affricated, hissing, uvular, consonants, etc.) nearly all of which he eliminates when he proceeds to the stage of a few words (...) that is to say, when he adopts the first semantic value. podem ser encontrados também nas primeiras palavras produzidas pelas crianças, principalmente nas onomatopéias. Além disso, contrariamente à existência do período de silêncio, pesquisadores observam que o balbucio continua até quando se inicia a produção dos primeiros itens lexicais (JUSCZYK, 1997).

Menn (1983) aponta três diferentes tipos de produções encontradas no período de transição do balbucio para as primeiras palavras: sound play, proto-palavras e balbucio modulado. $\mathrm{O}$ balbucio modulado se refere ao uso de sequência de sons que mostram algum significado através do contorno entonacional. No sound play, não há nenhuma indicação da relação entre a produção da criança e o significado. Já as proto-palavras são expressões articuladas com significado. Sabe-se que tais expressões possuem significado porque ocorrem em um contexto definido. É quando a criança tenta produzir uma palavra para um alvo determinado. Sobre o período de silêncio, Menn (1983) pontua que, a despeito da ênfase dada pela literatura, tal período é raro.

Assim, contrariando a hipótese de Jakobson, pesquisas apontam para a continuidade entre o balbucio e as primeiras palavras, não havendo, portanto, um período de silêncio e ruptura (VELLEMAN e VIHMAN, 2002). Além disso, o balbucio pode persistir, mesmo quando se inicia a produção dos primeiros itens lexicais. A seguir, apresentam-se pesquisas sobre a produção das primeiras palavras e sua relação possível com os períodos antecedentes.

\section{As primeiras palavras}

Pesquisas demonstram que a criança constrói o léxico inicial a partir da organização de padrões motores relacionados aqueles produzidos no período do balbucio (MACNEILAGE e DAVIS, 2000). De acordo com Velleman 
e Vihman (2002), as formas das primeiras palavras podem emergir de padrões vocais do balbucio. Assim, é observada uma semelhança entre as características do balbucio (como a ocorrência da reduplicação silábica e a produção de consoantes bilabiais) e as características dos primeiros itens lexicais. De acordo Bauman-Waengler (1996), os primeiros itens lexicais e o balbucio são tão parecidos que, por vezes, torna-se difícil diferenciá-los. Evidência para isso é a dificuldade metodológica que o pesquisador encontra na identificação de palavras produzidas pela criança, nos períodos bastante iniciais de aquisição da linguagem.

Por outro lado, há algumas diferenças entre a forma sonora das primeiras palavras e o balbucio. Conforme Vihman (1996), as consoantes labiais, por exemplo, são mais frequentes no balbucio do que nas primeiras palavras. Já as fricativas são menos frequentes no balbucio. Portanto, há influência do balbucio na produção lexical inicial, ou seja, as primeiras palavras são semelhantes às formas que ocorrem no balbucio. Porém, há também indícios de uma mudança na ocorrência de padrões sonoros específicos, quando o objetivo é produzir palavras (e não sequências de sons). Isso significa que ocorre uma mudança quando a criança produz palavras (unidades com a relação entre forma e significado) e sequência de sons aparentemente sem significação.

O período das primeiras 50 palavras é também conhecido como o período em que a criança se expressa através de uma única palavra por enunciado (single-word period). Ferguson e Farwell (1975), em um artigo clássico sobre o desenvolvimento fonológico inicial, propuseram analisar a produção das consoantes e vogais, como unidades válidas da fonologia infantil. Porém, os autores acabaram por concluir que a primeira fase de aquisição fonológica é baseada na palavra. Pesquisas que analisam a forma fonética das palavras nesse período observam que algumas crianças desenvolvem um padrão de produção (template), que será explicado a seguir.
Vihman e Miller (1988) apontam que o período de produção de uma única palavra por enunciado pode ser dividido em dois pontos principais, considerando as características fonéticas das palavras e o número de palavras produzido: o primeiro período, chamado 4-word-point (período das 4 palavras - earlywords), e o segundo período, chamado 25-wordpoint (período das 25 palavras - later words). A definição dos períodos é baseada em um recurso metodológico de identificação do número de palavras produzidas pela criança em interação espontânea com a mãe, em 30 minutos de gravação, comparado a uma lista oferecida pela mãe das palavras que a criança produz no dia a dia. Pesquisas mostram que há uma correlação entre o número de palavras produzido em uma sessão de gravação e as amostras de diário (VIHMAN, 1996; KEREN e PORTNOY, et al. 2009). Como é difícil definir o número de palavras que uma criança é capaz de produzir, já que não é possível acompanhá-la em diferentes momentos e contextos situacionais, esse é um recurso metodológico útil na definição do tamanho do vocabulário da criança. Apresenta-se, a seguir, a descrição dos dois períodos.

a) Primeiras palavras Early Words - Nesse momento, a criança produz, em uma sessão de 30 minutos de gravação, por volta de 4 palavras. Já de acordo com o relato da mãe, ela produz aproximadamente 8 palavras. As palavras que a criança produz, nesse período, o qual segue o balbucio, podem evidenciar duas características peculiares:

- São produzidas de forma bastante acurada. O termo "acurado" aqui deve ser compreendido de forma relativa, pois é utilizado considerando-se o ponto do processo de desenvolvimento em que a criança se encontra, pois ela ainda possui um repertório restrito de produção de palavras. Acurado, portanto, não significa idêntico, mas sim próximo ao alvo adulto. A expressão "alvo adulto" diz respeito 
ao repertório linguístico utilizado por aqueles que já adquiriram a linguagem e que, de certa forma, serve como "modelo" do que a criança almeja produzir.

- São selecionadas do alvo adulto. Ao produzir palavras "acuradas" a criança utiliza uma estratégia de seleção que é a segunda característica das palavras desse primeiro ponto de desenvolvimento. As palavras são geralmente selecionadas no alvo adulto. Assim, a criança tenta apenas uma pequena gama de palavras dentre as amplas possibilidades que a língua lhe oferece. Vihman e Croft (2007) apontam que, em geral, as formas preferidas seriam os tipos silábicos CVCV e VCV. Ferguson e Farwell (1975) observam que a criança seleciona no alvo adulto itens que estão de acordo com sua habilidade articulatória e, por isso, os produz adequadamente. Há, no entanto, controvérsias sobre a intencionalidade da criança ao selecionar as palavras no alvo adulto.

b) Últimas palavras Later Words - Nesse momento a criança tem, no seu vocabulário de produção de acordo com a mãe, aproximadamente, 50 palavras. Em uma sessão de 30 minutos de gravação, a criança produz 25 palavras, em média. Com o aumento do vocabulário, a acuracidade observada no período de produção das 4 palavras (4-word-point) pode se tornar menos evidente. Ou seja, em um segundo momento, a forma fonética das palavras produzidas pelas crianças podem se tornar distante daquela produzida pelo adulto. É, nesse período, que surgem os "padrões" (também chamados esquemas-templates). Velleman e Vihman (2002, p. 9) explicam o que é um padrão:

Um padrão é uma produção fonética abstrata ou esquemática que integra alvos salientes da palavra ou frases produzidas pelo adulto aos padrões vocais comuns da própria criança. Podem emergir das palavras-alvo mais frequentemente produzidas pela criança, com base nas formas fonéticas existentes da criança, e da adaptação de alvos menos estritamente selecionados para se encaixar ao padrão. (Tradução livre) ${ }^{2}$

Os padrões podem surgir, para algumas crianças, quando o vocabulário consta de, aproximadamente, 25 palavras ou mais (VIHMAN; VELLEMAN, 2000; VELLEMAN; VIHMAN, 2002). Nesse período, as palavras podem tornar-se menos acuradas e mais similares entre si. É como se houvesse uma modelo no qual as palavras fossem baseadas (wordrecipe - "receita de palavra"). Até mesmo as palavras inicialmente produzidas de forma bastante semelhante ao alvo adulto podem regredir para se adaptar ao esquema típico das crianças. É observada a utilização de duas estratégias principais na produção das palavras, nesse período:

1) a adaptação das formas do adulto para que se encaixem ao esquema seguido pela criança,

2) o evitamento de formas não acessíveis. Isto é, ou a criança não produz palavras que não se encaixem ao seu esquema de produção ou utiliza mecanismos de adaptação. Portanto, a seletividade fonológica que operou no primeiro ponto continua a atuar.

Este artigo foca, especificamente, no período chamado earlywords em transição para laterwords. Ou seja, procura-se refletir sobre o período de produção inicial das palavras para a consolidação e ampliação lexical, quando a criança já consegue produzir duas palavras ou mais por cada enunciado.

2 "A template is an abstract or schematic phonetic production pattern that integrates salient adult word or phrase targets and the child's own most common vocal patterns. It can be taken to emerge from target words frequently attempted by the child on the basis of the child's existing phonetic forms and from adaptation of less narrowly selected target words to fit the pattern." 


\section{Metodologia}

Este estudo é composto por dados de produção inicial de quatro crianças monolíngues, sendo três do sexo masculino e uma do sexo feminino. As crianças selecionadas não foram submetidas à terapia de fala, nem apresentaram relato de problemas de audição (informação obtida com os responsáveis). ${ }^{3}$

Como o objetivo deste artigo é discutir o desenvolvimento inicial a partir da análise da forma fonética de algumas das primeiras palavras, os sujeitos selecionados estavam em um período bastante inicial de aquisição da fala. Cogitou-se, em um primeiro momento, selecionar as crianças pela idade. Porém, sabe-se que há grande variação nos padrões de aquisição entre crianças de uma mesma idade (VIHMAN, 1996). Assim, optou-se por considerar o critério do número de palavras produzidas e não a idade. De acordo com Vihman (2009 p. 11):

Para as crianças que aprendem a mesma língua e especificamente para crianças que aprendem diferentes línguas, é importante usar o nível de desenvolvimento em vez da idade como base para comparação, uma vez que as crianças diferem de forma dramática, neste período, na velocidade de aprendizado lexical. (Tradução livre) ${ }^{4}$

Vihman e Miller (1988) apontam que deve ser considerado o número de palavras reportado pela mãe para se ter uma ideia do ponto de desenvolvimento lexical no qual se encontra a criança. Assim, considerando esse estudo, foram selecionadas crianças cujos pais relataram por volta $20 \mathrm{a}$ 25 palavras, com base em uma lista de palavras, fornecida pela mãe contendo

\footnotetext{
3 Aprovado no Comitê de ética da UFMG (CAAE - 0517.0.203.000-06).
4 "For children learning the same language and especially for children learning different languages we have found it important to use developmental level rather than age as the basis for comparison, since children differ so dramatically in this period in their rate of lexical learning."
}

todas as palavras que ela tivesse conhecimento que o filho/a produzia. Isso porque essas crianças estariam em um momento inicial de produção lexical.

Um levantamento das palavras produzidas por cada indivíduo foi realizado com os pais ou responsáveis, os quais forneceram uma lista de palavras as quais tinham conhecimento de que o filho seria capaz de produzir. Levando-se em conta os critérios acima, definiram-se os quatro informantes. Na Tabela 1, apresenta-se o informante, a idade na sessão 1 e o número de palavras produzido.

Tabela 1 - Informantes, idade e número de palavras na primeira sessão

\begin{tabular}{lccc}
\hline Informante & Idade & Gênero & $\begin{array}{c}\text { Número de palavras } \\
\text { durante a sessão }\end{array}$ \\
Paulo & $1: 11: 13$ & Masculino & 20 \\
Lucas & $1: 6: 8$ & Masculino & 8 \\
Laís & $1: 6: 24$ & Feminino & 12 \\
Gabriel & $1: 9: 21$ & Masculino & 11 \\
\hline
\end{tabular}

Fonte: Autora (2017).

Como se pode perceber, na Tabela 1, há diferença quanto à produção de palavras pelos quatro informantes. A produção de Paulo não corresponde à metade da produção referida pela mãe a qual reportou exatamente 25 palavras (na sessão, ele produziu 20 palavras). Porém, vale lembrar que há uma grande diferença entre os quatro informantes, na interação com a mãe e com a própria pesquisadora, principalmente, na primeira sessão. A mãe de Paulo participou efetivamente da coleta de dados, deixando à disposição fotografias e objetos aos quais Paulo fazia referência no dia a dia. Embora 
haja certa diferença quanto ao número de palavras produzidas, pode-se dizer que todas as crianças estão no período das primeiras cinquenta palavras e produzem uma palavra por enunciado.

Observa-se, também, a diferença de idade entre os informantes, principalmente entre Paulo e os demais. Contudo, reitera-se que o critério foi o número de palavras produzido relatado pelos responsáveis e não a idade. As crianças foram, mensalmente, gravadas por 12 meses. Embora os dados deste artigo digam respeito às primeiras sessões, apresenta-se, abaixo, a Tabela 2 com a idade dos sujeitos ao longo do tempo de gravação.

Tabela 2 - Idade dos Informantes ao longo do tempo

\begin{tabular}{|c|c|c|c|c|c|c|c|}
\hline \multicolumn{2}{|c|}{ Paulo } & \multicolumn{2}{|c|}{ Lucas } & \multicolumn{2}{|c|}{ Laís } & \multicolumn{2}{|c|}{ Gabriel } \\
\hline Sessão & Idade & Sessão & Idade & Sessão & Idade & Sessão & Idade \\
\hline 1 & 1;11:13 & 1 & $1 ; 9.21$ & 1 & $1 ; 6: 8$ & 1 & $1 ; 07: 25$ \\
\hline 2 & $2 ; 00: 20$ & 2 & $1 ; 10.31$ & 2 & $1 ; 7: 10$ & 2 & 1;09:08 \\
\hline 3 & $2 ; 1: 28$ & 3 & $2 ; 0.2$ & 3 & $1 ; 8: 9$ & 3 & $1 ; 10: 6$ \\
\hline 4 & $2 ; 2: 20$ & 4 & $2 ; 1.2$ & 4 & $1 ; 9: 12$ & 4 & 1;11:03 \\
\hline 5 & $2 ; 3: 22$ & 5 & $2 ; 1.27$ & 5 & 1;10:10 & 5 & $2 ; 00: 06$ \\
\hline 6 & $2 ; 4: 21$ & 6 & $2 ; 2.26$ & 6 & 1;11:15 & 6 & $2 ; 01: 03$ \\
\hline 7 & $2 ; 5: 20$ & 7 & $2 ; 3 ; 29$ & 7 & $2 ; 0: 15$ & 7 & 2;02:07 \\
\hline 8 & $2 ; 6: 19$ & 8 & $2 ; 4.26$ & 8 & $2 ; 1: 16$ & 8 & 2;03:00 \\
\hline 9 & $2 ; 7: 20$ & 9 & $2 ; 5.27$ & 9 & $2 ; 2: 13$ & 9 & $2 ; 04: 03$ \\
\hline 10 & $2 ; 8: 18$ & 10 & $2 ; 6.28$ & 10 & $2 ; 3: 13$ & 10 & $2 ; 05: 05$ \\
\hline 11 & $2 ; 9: 22$ & 11 & $2 ; 7.26$ & 11 & $2 ; 4: 11$ & 11 & $2 ; 06: 05$ \\
\hline 12 & $2 ; 10: 20$ & 12 & $2 ; 8.25$ & 12 & $2 ; 5: 15$ & 12 & 2:07:02 \\
\hline
\end{tabular}

Fonte: Autora (2017).
As sessões de gravações ocorreram em um local com menor interferência possível de ruídos, no ambiente familiar, o que propiciou a produção de fala espontânea, evitando-se a repetição. As sessões foram gravadas em áudio e vídeo, com duração aproximada de 30 minutos. Foi utilizado um gravador digital DAT (Digital Áudio Tape - Sony TCD-D8), com um microfone de lapela unidirecional Sony. Foram utilizados brinquedos, livros e objetos pessoais da criança em interação com o cuidado e a pesquisadora. Depois de coletados, os dados foram identificados e transcritos foneticamente, com a ajuda do programa praat. Os dados foram submetidos a uma análise qualitativa, considerando os princípios da Whole-Word Phonology. A seguir, apresenta-se a análise das palavras iniciais produzidas por cada uma das crianças.

\section{Análise dos dados: a forma sonora das palavras iniciais}

Apresenta-se, nesta seção, a análise individual das formas das palavras de cada um dos quatro informantes. Esta análise, cuja unidade será a palavra, tem cunho qualitativo e baseia-se nos princípios já delineados nas seções anteriores, da Whole-Word Phonoloy - teoria que considera a análise do item lexical, enquanto unidade de aquisição.

\section{Informante 1: Paulo}

O informante Paulo produziu formas simples, em sua maioria reduplicadas. Ele apresenta o que se pode chamar de padrões e realiza adaptações para que as palavras se encaixem nestes padrões fonológicos. As principais adaptações que Paulo realiza nas palavras são: (1) omissão da sílaba (truncamento) e, nesse caso, a forma de produção resultante é CV, (2) reduplicação - que é a repetição da mesma sílaba - e (3) harmonia - que é a reduplicação parcial, conforme pode ser visto no Quadro 1, a seguir 
Quadro 1 - Produção inicial de Paulo

\begin{tabular}{|l|l||l|l|}
\hline Forma ortográfica & Forma da criança & Forma ortográfica & Forma da criança \\
\hline carro & 'ahu & neném & nẽ'nẽ \\
\hline (O) boi & u'boi & vovó & vo'vo \\
\hline água & 'abua & vovô & vo'vo \\
\hline acabou & a'bo & papai & pa'paI \\
\hline pão & 'pã & mamãe & ma'maI \\
\hline Paulo & 'pau & tartarua & ta'ta \\
\hline banho & 'bã & Luciana & Pu'?u \\
\hline bola & 'boa & Roseli & ?i'?i \\
\hline bolo & 'bo & Letícia & ta'ta te'ta \\
\hline Edmar & 'ma & copo & 'pop? \\
\hline bombom & bu'bũ & Silene & \\
\hline bumbum & & & \\
\hline
\end{tabular}

Fonte: Autora (2017).

No total dos itens produzidos, a maioria são itens $\operatorname{CVCV}(V)$, em que (V) significa a ocorrência de um glide opcional. De fato, esse é o padrão mais sólido nos dados de Paulo. As formas CV e CVCV podem representar um resquício do padrão combinatório do balbucio canônico, qual seja a produção ritmada de sílaba CV (VIHMAN, 1993; VIHMAN, VELLEMAN, 2000; OLIVEIRA-GUIMARÃES, 2013; BAIA,2013). A ocorrência da harmonia consonantal na fala da criança tem chamado a atenção de pesquisadores, principalmente, por não ser um processo comum na fala do adulto (VIHMAN, 1978; BERG, ULRICH, 2000). Chama atenção também o fato de ocorrer amplamente, sendo encontrada na aquisição de diversas línguas (VIHMAN, 1978).

As formas reduplicadas são, muitas vezes, tão díspares do modelo adulto, que é difícil analisá-las em termos de processos fonológicos, considerando os segmentos um a um. Não é possível estabelecer uma correspondência sistemática e linear entre as formas da criança e do adulto em produções do tipo [ta'ta] para "Letícia", por exemplo. Tal fato oferece suporte para Whole-Word Phonology (WATERSON, 1971; FERGUSON, FARWELL, 1975; VIHMAN, 1996). A impossibilidade de se analisarem as formas da criança de uma maneira direta e linear com o alvo adulto é um dos argumentos de Ferguson e Farwell (1975), para postular que a palavra, pelo menos nas fases iniciais, é a unidade de aquisição. Assim, pode-se dizer que criança não inicia a aquisição da linguagem através de unidades como segmentos ou fonemas. A criança aprende rotinas articulatórias ou padrões em palavras específicas. Daí a importância do item lexical, que é aprendido, gradualmente, um a um.

\section{Informante 2: Lucas}

As palavras produzidas por Lucas estão apresentadas no Quadro 2 e, inicialmente, possuem uma característica peculiar do momento o qual Vihman e Veeleman (2000) denominamearly words, ou palavras iniciais, que se refere ao ponto em que a criança produz, no mínimo, 4 palavras espontaneamente em uma sessão de gravação, o chamado 4-word-point (ponto das 4 palavras). Nesse momento, as palavras produzidas são, em sua maioria, selecionadas. Ou seja, a criança usa estratégias para "captar", na fala 
do adulto, apenas palavras que é capaz de produzir (FERGUSON, FARWELL, 1978).

Quadro 2 - Palavras produzidas por Lucas

\begin{tabular}{|c|c|}
\hline Forma ortográfica & Produção da criança \\
\hline 1. Cacá & 'ta \\
\hline 2. esse & 'ef \\
\hline 3. Gisele & zi'zi \\
\hline 4. mamãe & u'عmai, mã'mãı \\
\hline 5. nãnão & nã'nãu \\
\hline 6. oi & 'or \\
\hline 7. papai & pa'par \\
\hline 8. Zizi (apelido) & 3i'3I \\
\hline
\end{tabular}

Fonte: Autora (2017).

Diferentemente de Paulo, observa-se que Lucas utiliza palavras-chave, que parecem, de fato, selecionadas do alvo adulto. Ou seja, Lucas realiza poucas adaptações nas palavras produzidas. Tal fato reforça o papel ativo da criança na aquisição e levanta questionamentos a respeito de sua intencionalidade ao fazer a seleção de palavras no vocabulário.

\section{Informante 3: Laís}

Os dados de Laís são apresentados no Quadro 3 e indicam que ela não segue um padrão específico, mas tenta diferentes formas, como por exemplo, a sequência "é palhaço", a qual é produzida com um padrão de produção complexo: [عna'lapsuki]. Além da produção de formas relativamente complexas, Laís mostra grande variação na produção de uma mesma palavra.
Por exemplo, a palavra "aqui ó" é pronunciada, ora com o cancelamento da consoante velar, ora com a oclusiva alveolar no lugar da consoante velar, ora como a forma alvo.

Quadro 3 - Palavras produzidas por Laís

\begin{tabular}{|c|c|c|}
\hline \multicolumn{2}{|c|}{ Forma ortográfica } & \multirow{2}{*}{\begin{tabular}{|l} 
Produção da criança \\
bo \\
\end{tabular}} \\
\hline 1. & acabou & \\
\hline 2. & aí & $a^{\prime} i$ \\
\hline 3. & aquió & a'i, ati'っ, aki'॰, a'kə, titi'॰ \\
\hline 4. & $\mathrm{au}$ au au & auauau \\
\hline 5. & Bruninho & 'nin \\
\hline 6. & chave & 'tapa \\
\hline 7. & dado & 'dat ${ }^{\mathrm{h}}$ \\
\hline 8. & É & $\varepsilon$ \\
\hline 9. & élá & E'la \\
\hline 10. & Épalhaço & عna'lapsuki \\
\hline 11. & Épão & ع'pã pãu \\
\hline 12. & esse & 'e, 'ef \\
\hline 13. & Eulinho & ĩu \\
\hline 14. & gato & 'gaku \\
\hline 15. & lá & 'la \\
\hline
\end{tabular}

Fonte: Autora (2017).

Além da não aplicação ou da não consistência na produção de determinado padrão, e da variabilidade na produção de uma mesma palavra, outro fato que chama a atenção é a produção de uma vogal com uma espécie de âncora, como $[\varepsilon]$, ou [o], o que pode ser um resquício das vocalizações 
presentes no balbucio ou mesmo uma tentativa já de chamar a atenção do interlocutor para o que está dizendo.

\section{Informante 4: Gabriel}

O que mais chama atenção nos dados de Gabriel é a grande variabilidade na produção dos itens lexicais. A variação é tanto verificada entre os itens lexicais, que não demonstram seguir uma forma esquemática única, quanto em um mesmo item lexical, como o item "palhaço", por exemplo, que é produzido de 5 formas distintas.

Quadro 4 - Palavras produzidas por Gabriel

\begin{tabular}{|c|c|}
\hline Forma ortográfica & Produção da criança \\
\hline abriu & iu'iu, a'iu \\
\hline carrinho & ka'ĩ \\
\hline girafa & 'afa \\
\hline girafinha & a'fi $(2 x)$ 'ati \\
\hline grandão & tã'tãu \\
\hline jacaré & 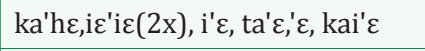 \\
\hline mamãe & mã'mãı, mãı, mmãı \\
\hline não & 'nã(2x), 'nãu(3x) \\
\hline outro & 'oth $(8 x)$ \\
\hline palhacinho & pa'sĩ(2x), a'sĩ paa'tĩ \\
\hline palhaço & 'pas, pa'as, pa'asu, asu'asu, pa'ati \\
\hline pato & 'path \\
\hline peixinho & 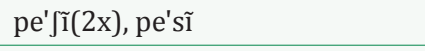 \\
\hline peteca & 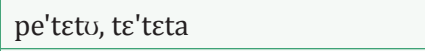 \\
\hline piu-piu & bi'biv \\
\hline sofá & 'fa \\
\hline
\end{tabular}

Fonte: Autora (2017).
Essa grande variabilidade na produção inicial foi também observada nos dados da informante Laís, o que sugere que:

1) alguns informantes, nos momentos iniciais de aquisição fonológica, utilizam estratégias como a aplicação de um padrão específico na produção das palavras, o que permitiria a expansão do vocabulário com a utilização de uma forma fonética única (VIHMAN, CROFT, 2007). Esse seria o caso de Paulo e Lucas.

2) outros informantes são bastante "corajosos" na sua produção inicial. Tentam alvos aparentemente difíceis e são altamente variáveis, como no caso de Laís e Gabriel.

Outra característica importante dos dados de Gabriel é o truncamento (ou omissão de sílabas), como na palavra "sofá" [fa], "girafa" ['afa], "girafinha" [a'fi] "jacaré" [i'c] "palhacinho" [a'sĩ], entre outros. Assim, Gabriel aplica um processo diferente de Laís, que, geralmente, adiciona sílabas, às palavras. Em Gabriel, nota-se uma tendência ao encurtamento. De acordo com Taelman (2004), no truncamento, há um forte condicionamento prosódico, mas também a influência secundária de fatores como sonoridade do onset (consoante inicial) e a presença ou ausência de coda (posvocálica). Nos dados de Gabriel, observa-se uma tendência àpreservação da sílaba tônica.

A reduplicação e harmonia são também verificadas, como em "jacaré" [i $\left.\varepsilon^{\prime} i \varepsilon\right]$ ou [ $\varepsilon^{\prime} \varepsilon$ ], "abriu" [iu'iu], "grandão" [tã'tãu]. Porém, essas formas aparecem em competição com outras formas, o que não permite afirmar que Gabriel utiliza um "padrão". Interessante notar que a forma "grandão" continua sendo produzida com a harmonia consonantal até a sessão 12, não apresentando variação. 


\section{Considerações finais}

Neste artigo, apresentou-se uma análise da produção inicial de quatro informantes. Todos esses estariam no período de produção de uma palavra por enunciado e contavam, de acordo com o relato dos pais, com a produção de 25 palavras, aproximadamente. Assim, foi possível refletir sobre a forma sonora das palavras em período anterior à produção de enunciados e sentenças.

De modo geral, observou-se que algumas formas são resquícios do balbucio, como CVCV. Uma das crianças apresentou uma adaptação maior ao alvo, utilizando padrões motores específicos, o que sugere que alguns sujeitos apresentem maior relação com as formas de reduplicação presentes no balbucio do que outros sujeitos no mesmo período de aquisição. Vale lembrar também que tais formas podem estar presentes na produção do adulto dirigida à criança. Por outro lado, houve dados que demostraram maior ocorrência de formas selecionadas do alvo adulto, ou seja, produções bastante acuradas e que, já no alvo adulto, apresenta um padrão que se "encaixa" ao da criança. Além desses casos, observou-se que uma das crianças tentou formas diversas, sendo verificada a variação intrapalavra.

Os dados apresentados evidenciam os princípios da teoria da WholeWord Phonology, pela qual se propõe que os dados de produção inicial da criança são mais bem avaliados considerando o item lexical como um todo e não processos fonológicos específicos. Apontam também a necessidade de um olhar individualizado e específico para fala da criança enquanto um sujeito que encontra alternativas diversificadas para construir seu caminho em direção à aquisição da linguagem.

\section{Referências}

BAIA, Maria Fatima Almeida. Os templates no desenvolvimento fonológico: o caso do português brasileiro. Tese (Doutorado em Linguística) - FFLCH/USP. 2013.

BAUMAN-WAENGLER, Jacqueline. Desenvolvimento fonológico normal. In: LOWE, Robert. Fonologia: Avaliação e intervenção: aplicações na patologia da fala. Tradução Marcos A. G. Domingues. Porto Alegre: Artes Médicas, 1996. cap. 3, p. 33-65. [Título original: Phonology: assessment and intervention: applications in speech pathology.]

BERG, Tomas; SHADE, Urich. S. A local connectionist account of consonant harmony in child language. Cognitive Science, v. 24, p. 123-149, 2000.

FERGUSON, Charles A.; FARWELL, Carol. B. Words and sounds in early language acquisition. Language, v. 51, p. 419-439. 1975.

JAKOBSON, Roman. The sound laws of child language and their place in general phonology. In: LEOPOLD, W. F.; BAR-ADON, A. (Ed.). Child language: a book of readings. Englewood Cliffs: Prentice-Hall, 1971 [1939]. p. 75-82.

JUSCZYK, P.eter. The discovery of spoken language. Cambridge, Mass.: MIT Press, 1997.

KEREN-PORTNOY, Tamar, MAJORANO, Marinella; VIHMAN, Marilyn M. From phonetics to phonology: Theemergence of first words in Italian. Journal of Child Language, v. 36, p. 235-267, 2009.

LAMPRECHT, Regina; BONILHA, Giovana F. G.; FREITAS, Gabriela C. M.; MATZENAUER, Carmem Lúcia B.; MEZZOMO, Carolina L.; OLIVEIRA, Carolina C.; RIBAS, Letícia. P. Aquisição fonológica do português: perfil de desenvolvimento e subsídios para terapia. São Paulo: Artmed, 2004.

MENN, Lise Development of articulatory, phonetic, and phonological capabilities. In: DANS BUTTERWORTH, B. (Ed.). Language Production. London: Academic Press, 1983. v. 2, p. 3-49.

OLIVEIRA-GUIMARÃES, Daniela Mara Lima. Beyond early words: word template development in Brazilian Portuguese. In: VIHMAN, M. M.; KEREN-PORTNOY, Tamar. The Emergence of phonology: whole word approaches and cross-linguistic evidence. London: Cambridge university press, 2013. p. 291-316.

OLIVEIRA-GUIMARÃES, Daniela Mara Lima. Percurso da construção da fonologia pela criança: uma abordagem dinâmica. Tese (Doutorado em Linguística) - Universidade Federal de Belo Horizonte, Belo Horizonte, 2008. 
TAELMAN, Helena. Syllable omissions and additions in Dutch child language: an inquiry into the function of rhythm and the link with grammar. PhD dissertation. 2004. 302 p.

VELLEMAN, Shelley.; VIHMAN, Marilyn May. Whole-word phonology and templates. Language speech and hearing services in schools, v. 23, p. 9-23, 2002.

VIHMAN, Marilyn May. Consonant harmony: its scope and function in child language. In: GREENBERG, Joseph Harold; FERGUSON, Charles; MORAVCSIK, Edith (Ed.). Universals of Human Language, 2. Stanford: Stanford University Press, 1978. p. 281-334.

VIHMAN, Marilyn May. Phonological development: the origins of language in the child. Cambridge: Blackwell publishers, 1996. 312 p.

VIHMAN, Marilyn May. Variable paths to early word production. Journal of Phonetics, v. 21, p. 61- 81, 1993.

VIHMAN; Marilyn May; MILLER, R. Words and babble at the threshold of lexical acquisition. In: DANS SMITH, Michael; LOCKE, John (Ed.). The emergent lexicon. New York: Academic Press. 1988.

VIHMAN, Marilyn May. Word learning and the origins of phonological system. In: FOSTERCOHEN, S. (Ed.). Advances in language acquisition. Luton: Macmillan, 2009.

VIHMAN, Marilyn May; VELLEMAN, Shelley. Phonetics and the origins of phonology. In: BURTON- ROBERTS, N.; CARR, P.; DOCHERTY, G. (Ed.). Phonological knowledge: Conceptual and empirical issues. Oxford: Oxford University Press, 2000. p. 305-339.

VIHMAN, Marilyn May; CROFT, William. Phonological development: Toward a 'radical' templatic phonology. Linguistics, v. 45, p. 683-725. 2007.

WATERSON, Natalie. Child phonology: a prosodic view. Journal of Linguistics, v. 7, p. 179-211, 1971.

Recebido em: 25/01/201

Aceito em: 04/06/2017 